\title{
Thickening of the Left Atrial Wall Shortly After Radiofrequency Ablation Predicts Early Recurrence of Atrial Fibrillation
}

\author{
Miki Yokokawa, MD; Hiroshi Tada, MD; Keiko Koyama, MD; Toshihiko Ino; \\ Shigeto Naito, MD; Shigeru Oshima, MD; Koichi Taniguchi, MD
}

\begin{abstract}
Background: Inflammatory responses following atrial fibrillation (AF) ablation may aggravate arrhythmogenic activity and cause an early recurrence of $A F$ (ERAF).

Methods and Results: In 56 patients who underwent circumferential pulmonary vein ablation (CPVA) for paroxysmal AF, cardiovascular magnetic resonance imaging (CMRI) was evaluated in 5 subdivided left atrial (LA) regions before the CPVA and at 1 day and 1 month after. At a mean of $7 \pm 10$ days after the CPVA, 23 patients $(41 \%)$ developed 1 or more episodes of AF (ERAF group), while 33 patients $(59 \%)$ remained free from AF during the first month of follow up (no-ERAF group). LA wall thickness increased 1 day after the CPVA in both groups, as demonstrated by high T2-weighted signal. The LA roof thickness and its increase, however, were greater in the ERAF group than in the no-ERAF group $(P<0.05)$. Regions of delayed enhancement $(D E)$ were also frequently detected in both groups, but the total number of $D E$ regions did not differ between the 2 groups. The thickening of the LA wall associated with a high T2-weighted signal resolved within 1 month. No significant difference between the 2 groups was found in any of the CMRI parameters before or 1 month after CPVA.
\end{abstract}

Conclusions: Thickening of the LA roof shortly after CPVA may predict an ERAF. (Circ J 2010; 74: 15381546)

Key Words: Atrial fibrillation; Edema; Magnetic resonance imaging; Radiofrequency ablation

C ircumferential pulmonary vein ablation (CPVA) to eliminate paroxysmal atrial fibrillation (AF) consists of delivering radiofrequency (RF) energy to each pulmonary vein $(\mathrm{PV})$ antrum to electrically isolate the PVs. ${ }^{1}$ AF recurrences within 1 month after CPVA, however, have been reported in up to $40 \%$ of cases. ${ }^{2,3}$ Because myocardial edema develops early after the ablation procedure, ${ }^{4}$ the acute inflammation caused by the thermal injury may transiently exacerbate the arrhythmogenic activity of the PV. ${ }^{5}$

\section{Editorial $p 1524$}

Recent advances in delayed enhancement (DE) cardiovascular magnetic resonance imaging (CMRI) have enabled the study of atrial scar tissue, mechanisms of RF ablation, and of the relationship between RF ablation and the resultant anatomical and histological changes. ${ }^{6,7} \mathrm{~A}$ few studies reported acute changes in the left atrial (LA) wall using electron-beam computed tomography ${ }^{4}$ or intracardiac echo imaging. ${ }^{8}$ To the best of our knowledge, however, no studies have examined those changes using CMRI. The purpose of the present study was therefore to use CMRI to examine the relationship between the early changes that occur in the LA wall after RF ablation and recurrences of AF.

\section{Study Population}

The present study included 56 patients (mean age $=60 \pm 11$ years, 42 men) presenting with symptomatic, drug-refractory $\mathrm{AF}$, who underwent $\mathrm{CMRI}$ and $\mathrm{RF}$ catheter ablation between October 2006 and March 2008 (Table 1). All patients had paroxysmal AF, which was defined as AF episodes that spontaneously terminated and lasted for $>30 \mathrm{~s}$ and $<7$ days during treatment with anti-arrhythmic drugs. CMRI was performed 1 day before, 1 day after and 1 month after the CPVA. Patients were excluded from this analysis if they (1) had contraindications to CMRI; (2) had undergone previous cardiac surgery or catheter ablation of AF; or (3) were not in sinus rhythm during both the pre- and post-procedural CMRI assessments. Ethics

Received January 12, 2010; accepted March 18, 2010; released online June 19, 2010 Time for primary review: 15 days

Division of Cardiology (M.Y., S.N., S.O., K.T.), Division of Radiology (K.K., T.I.), Gunma Prefectural Cardiovascular Center, Maebashi; and Tsukuba University Hospital, Graduate School of Comprehensive Human Sciences, University of Tsukuba, Tsukuba (H.T.), Japan

Mailing address: Hiroshi Tada, MD, Cardiovascular Division, Institute of Clinical Medicine, Graduate School of Comprehensive Human Sciences, University of Tsukuba, 1-1-1 Tennodai, Tsukuba 305-8575, Japan. E-mail: htada@md.tsukuba.ac.jp

ISSN-1346-9843 doi:10.1253/circj.CJ-10-0026

All rights are reserved to the Japanese Circulation Society. For permissions, please e-mail: cj@j-circ.or.jp 
A. Coronal
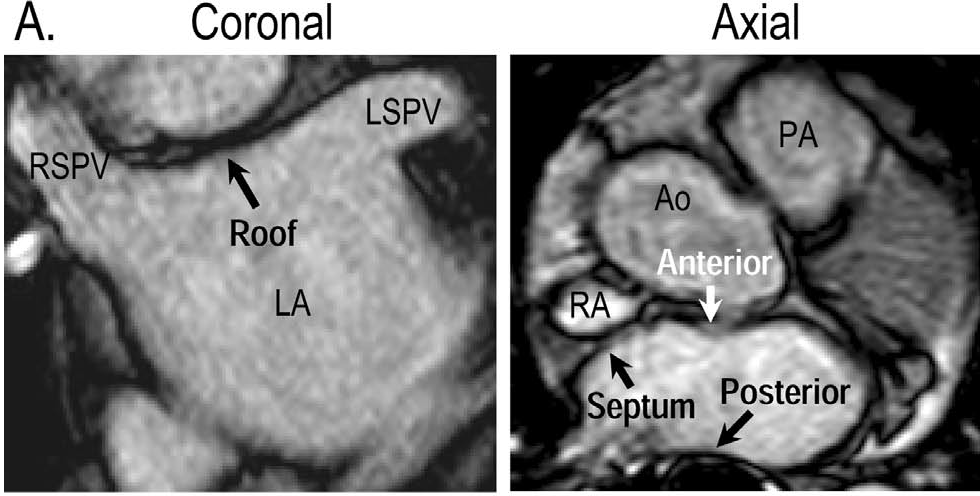

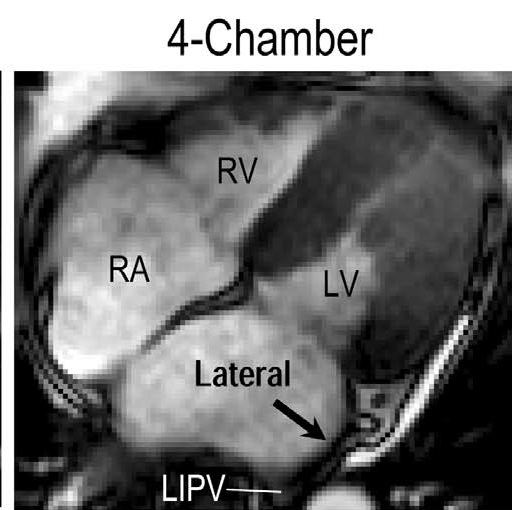

4-Chamber

B.

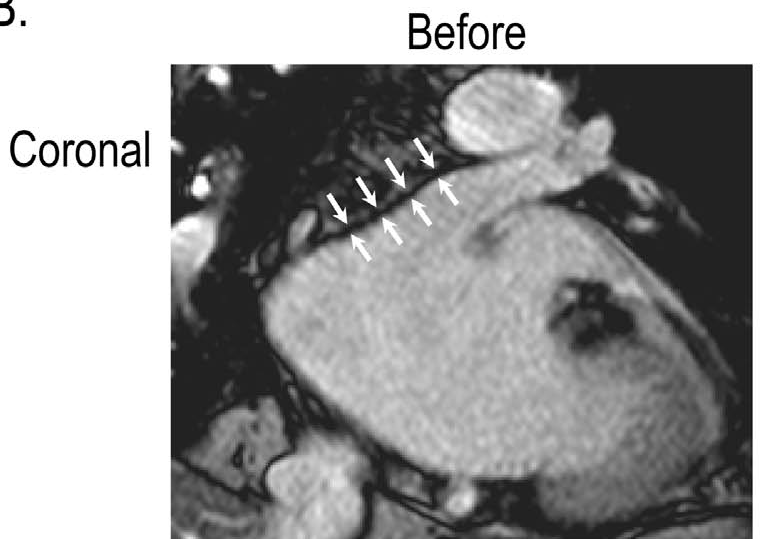

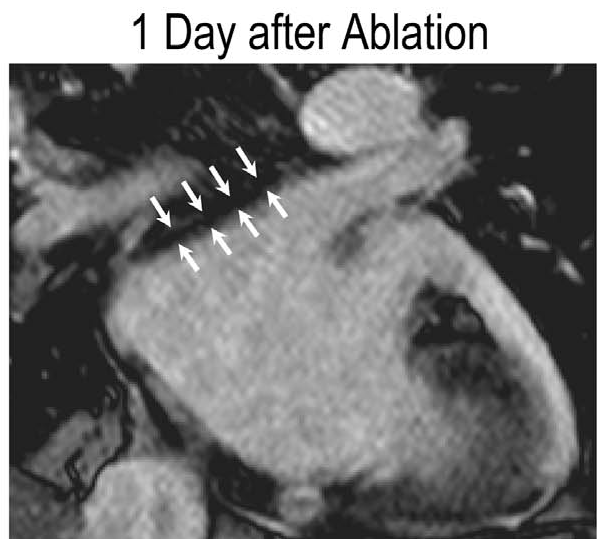

Figure 1. (A) Anterior, posterior and lateral walls, interatrial septum, and roof of the left atrium on cardiovascular magnetic resonance imaging. (B) Increase in the left atrial roof thickness (T1-weighted imaging) from $1.6 \mathrm{~mm}$ before, to $2.6 \mathrm{~mm} 1 \mathrm{day}$ after ablation (arrows). Ao, aorta; LIPV, left inferior pulmonary vein; LSPV, left superior pulmonary vein; LV, left ventricle; PA, pulmonary artery; RA, right atrium; $\mathrm{RSPV}$, right superior pulmonary vein; $\mathrm{RV}$, right ventricle.

approval was obtained from the Institutional Ethics Review Committee of Gunma Prefectural Cardiovascular Center and all patients granted informed, written consent to participate.

\section{Magnetic Resonance Imaging Data Acquisition}

CMRI was performed with a 1.5-T Achieva imaging scanner (Philips Medical System, Eindhoven, Netherlands) equipped with a Nova gradient and 5-element cardiac synergy coil. The LA images were acquired using breath-hold balanced, steady-state free precession cine CMRI, performed in the 4-chamber long-axis, 2-chamber sagittal, axial and coronal projections, with retrospective electrocardiographic (ECG) gating to obtain 30 phases/R-R interval. The LA was completely covered with 16-22 transverse slices acquired during retrospective ECG-gated, cine pulse sequences. All images were acquired during an expiratory breath-hold (1-2 slices per breath-hold depending on the patient's heart rate and tolerance) and were used to study the LA morphology throughout the cardiac cycle. Typical scan characteristics were as follows: $6-\mathrm{mm}$ slice thickness, no gap between slices, $2.0 \times 2.0 \mathrm{~mm}$ pixel size, $2.56 / 1.03 \mathrm{~ms}$ repetition (TR)/echo time (TE), and 15 views/segment.

We applied a T2-weighted triple inversion-recovery sequence $[\mathrm{TR}=2 \times \mathrm{R}-\mathrm{R}$ interval; $\mathrm{TE}=65 \mathrm{~ms}$; inversion time $(\mathrm{TI})=$ $140 \mathrm{~ms}$ ] to study the myocardium, as described in detail elsewhere. ${ }^{9}$ Briefly, a pair of slice-selective and non-selec- tive 180 inversion pulses were applied to null the blood signal and a third inversion pulse was applied to null the fat signal. DE-CMRI was acquired $15 \mathrm{~min}$ after the injection of a $0.15-\mathrm{mmol} / \mathrm{kg}$ bolus of Magnevist gadolinium diethylenetriamine pentaacetic acid contrast material (Bayer Schering Pharma, Berlin, Germany), using a respiration-navigated, ECGgated, gradient echocardiographic pulse sequence. Typical acquisition settings were as follows: TR/TE $=4.0 / 2.0 \mathrm{~ms}$ and $\mathrm{TI}=230-270 \mathrm{~ms}$. ECG-gating was used to acquire a small subset of phase-encoding views during the diastolic phase of the LA cycle. The time interval between the peak of the $\mathrm{R}$ wave and onset of the data acquisition was defined using the LA cine images. Fat saturation was used to suppress the fat signal. The 2.0-ms TE was chosen to (1) keep fat and water out of phase from one another; and (2) decrease the signal intensity of the partial volume fat-tissue voxels, improving the definition of the LA wall boundary.

The TI for the DE-CMRI was obtained for each patient by visual inspection of the preliminary acquisition of a singleslice multi-TI series. The typical duration of DE-CMRI was 5-10 min, depending on the patient's respiratory and heart rates. If the TI at first acquisition was not optimal or marked motion artifact was observed, the scan was repeated.

\section{Catheter Ablation}

All anti-arrhythmic medications were discontinued for 5 half- 


\begin{tabular}{|c|c|c|c|c|}
\hline & $\begin{array}{l}\text { All patients } \\
(n=56)\end{array}$ & $\begin{array}{l}\text { Patients with } \\
\text { ERAF }(n=23)\end{array}$ & $\begin{array}{l}\text { Patients with no } \\
\text { ERAF }(n=33)\end{array}$ & $P$ value \\
\hline \multicolumn{5}{|l|}{ Baseline demographics } \\
\hline Age (years) & $60 \pm 11$ & $58 \pm 12$ & $61 \pm 10$ & 0.21 \\
\hline Men, n (\%) & $42(75)$ & $13(57)$ & $29(88)$ & $<0.01$ \\
\hline Duration of atrial fibrillation (years) & $5.0 \pm 4.1$ & $4.6 \pm 3.7$ & $5.2 \pm 4.5$ & 0.71 \\
\hline Body mass index $\left(\mathrm{kg} / \mathrm{m}^{2}\right)$ & $25 \pm 4$ & $26 \pm 5$ & $24 \pm 3$ & 0.08 \\
\hline Hypertension, n (\%) & $11(20)$ & $7(26)$ & $4(12)$ & 0.29 \\
\hline Structural heart disease, $\mathrm{n}(\%)$ & $4(7)$ & $2(9)$ & $2(6)$ & 1.00 \\
\hline Anti-arrhythmic drugs, $\mathrm{n}(\%)$ & $56(77)$ & $18(78)$ & $25(76)$ & 1.00 \\
\hline Left atrial size $(\mathrm{mm})$ & $40 \pm 5$ & $40 \pm 5$ & $40 \pm 6$ & 0.80 \\
\hline Left ventricular ejection fraction (\%) & $66 \pm 9$ & $67 \pm 8$ & $66 \pm 9$ & 0.59 \\
\hline \multicolumn{5}{|l|}{ Procedural variables } \\
\hline Total duration of RF energy applications (min) & $43 \pm 10$ & $43 \pm 10$ & $42 \pm 10$ & 0.77 \\
\hline Total amount of RF energy $\left(\times 10^{3} \mathrm{~J}\right)$ & $68 \pm 18$ & $73 \pm 13$ & $64 \pm 20$ & 0.12 \\
\hline Late recurrence of atrial fibrillation, $\mathrm{n}(\%)$ & $11(20)$ & $6(26)$ & $5(15)$ & 0.50 \\
\hline \multicolumn{5}{|l|}{ CMRI parameters } \\
\hline \multicolumn{5}{|l|}{$\Delta$ Wall thickness (mm) } \\
\hline Roof & $1.2 \pm 1.0$ & $2.0 \pm 1.0$ & $0.7 \pm 0.5$ & $<0.0001$ \\
\hline Anterior wall & $0.3 \pm 0.5$ & $0.3 \pm 0.3$ & $0.4 \pm 0.6$ & 0.71 \\
\hline Posterior wall & $0.2 \pm 0.3$ & $0.3 \pm 0.4$ & $0.2 \pm 0.3$ & 0.08 \\
\hline Septum & $0.3 \pm 0.4$ & $0.4 \pm 0.4$ & $0.3 \pm 0.3$ & 0.62 \\
\hline Lateral wall & $0.3 \pm 0.3$ & $0.4 \pm 0.3$ & $0.3 \pm 0.3$ & 0.86 \\
\hline$\Delta$ Total no. high-signal T2-weighted regions & $3 \pm 1$ & $3 \pm 1$ & $3 \pm 1$ & 0.16 \\
\hline$\Delta$ Total no. DE regions & $2 \pm 1$ & $2 \pm 2$ & $2 \pm 1$ & 0.59 \\
\hline
\end{tabular}

lives before the procedure, except for amiodarone, which was discontinued for at least 6 weeks. Via trans-septal catheterization, a 7F Lasso decapolar ring catheter (Biosense Webster, Diamond Bar, CA, USA) and Blazer II 5770T 7F quadripolar ablation catheter with an 8-mm distal electrode and deflectable tip (EP Technologies, Boston Scientific, San Jose, CA, USA) were inserted into the LA. After selective PV angiography, the Lasso catheter was positioned within $5 \mathrm{~mm}$ of the PV ostium. The bipolar electrograms were recorded digitally (EPMed Systems, Mount Arlington, NJ, USA) and filtered at a bandpass of 30-500 Hz. RF energy was delivered via the 8 -mm tip catheter at a maximum temperature of $55^{\circ} \mathrm{C}$ and maximum power of $30-35 \mathrm{~W}$, for $30 \mathrm{~s}$ at each site, approximately 10-20 mm outside each PV ostia. The duration of RF delivery was increased to $60 \mathrm{~s}$ at sites where changes in the PV activation sequence were observed and at the disconnection sites. The endpoints of the CPVA were (1) the creation of bidirectional conduction block between the atrium and the PVs, ascertained immediately and 30-60 min after a successful PV isolation; and (2) no induction of AF episodes lasting $>3 \mathrm{~min}$ by coronary sinus burst pacing to a cycle length of $180 \mathrm{~ms}$, during the infusion of iv isoproterenol at $1.0-3.0 \mu \mathrm{g} / \mathrm{min}$. Bidirectional conduction block was confirmed by (1) entrance block, demonstrated by the loss of all PV potentials; and (2) exit block, ascertained during sinus rhythm by failure to capture the LA when pacing from all bipolar pairs of electrodes on the Lasso catheter placed at the entrance of the PV. ${ }^{10}$

\section{Procedure}

The LA wall thickness was measured and the DE and T2weighted images of the LA wall were evaluated. The mea- surements were done in 5 LA anatomic regions: anterior wall, posterior wall, interatrial septum, roof (between the superior aspect of the left and right PVs) and lateral wall (Figure 1A). Calipers were used to measure the LA endocardial to epicardial thickness at the point of the largest LA dimension at the end of ventricular systole (Figure 1B). The difference in the LA wall thickness before vs after the CPVA was recorded and T2-weighted imaging of the LA wall evaluated semi-quantitatively. The acute changes after the ablation were defined as an increase in the LA wall thickness associated with a high T2-weighted signal not observed before the CPVA.

The definition of normal vs injured tissue was based on the distribution of the pixel intensities within the LA wall, with the lowest intensity defined as normal tissue. A region was classified as affected when the tissue's pixel intensity was $>2$ SD above the mean pixel intensity of normal tissue on both T2-weighted imaging and DE-CMRI. ${ }^{11,12}$ All images were analyzed by 2 observers, experienced in the interpretation of CMRI, who were unaware of the patient's clinical status; a third reviewer was consulted in the case of discordant interpretations.

\section{Post-Ablation Follow up}

All patients remained hospitalized and their rhythm was continuously monitored for approximately 1 week after the ablation procedure. The patients who were treated with an antiarrhythmic drug before the procedure remained on the same drug regimen for 3 months thereafter. After their discharge from hospital, the patients returned to the ambulatory cardiology department 2 weeks later and monthly thereafter. At 2 weeks, 1 month and at 6 months after CPVA, 12-lead or 


\begin{tabular}{|lccc|}
\hline Table 2. CMRI Parameters vs Time After CPVA in All Patients (Mean \pm SD) & & \\
Left atrial wall thickness (mm) & Before CPVA & $\begin{array}{c}\text { 1 day after } \\
\text { CPVA }\end{array}$ & $\begin{array}{c}\text { 1 month after } \\
\text { CPVA }\end{array}$ \\
Roof & & & $1.6 \pm 0.5^{\dagger}$ \\
Anterior wall & $1.5 \pm 0.4$ & $2.8 \pm 1.2^{*}$ & $1.7 \pm 0.3^{\dagger}$ \\
Posterior wall & $1.8 \pm 0.4$ & $2.1 \pm 0.5^{*}$ & $1.6 \pm 0.3^{\dagger}$ \\
Septum & $1.6 \pm 0.3$ & $1.8 \pm 0.4^{*}$ & $1.6 \pm 0.2^{\dagger}$ \\
Lateral wall & $1.6 \pm 0.3$ & $2.0 \pm 0.4^{*}$ & $1.6 \pm 0.3^{\dagger}$ \\
Total no. high-signal T2-weighted regions & $1.6 \pm 0.3$ & $2.0 \pm 0.3^{*}$ & $1.1 \pm 1.0^{\dagger}$ \\
Total no. DE regions & $0 \pm 0$ & $2.7 \pm 0.7^{*}$ & $4.6 \pm 0.8^{*}$ \\
\hline
\end{tabular}

${ }^{*} \mathrm{P}<0.001$ vs before CPVA; $\mathrm{P}<0.001$ vs 1 day after CPVA.

Abbreviations see in Table 1.

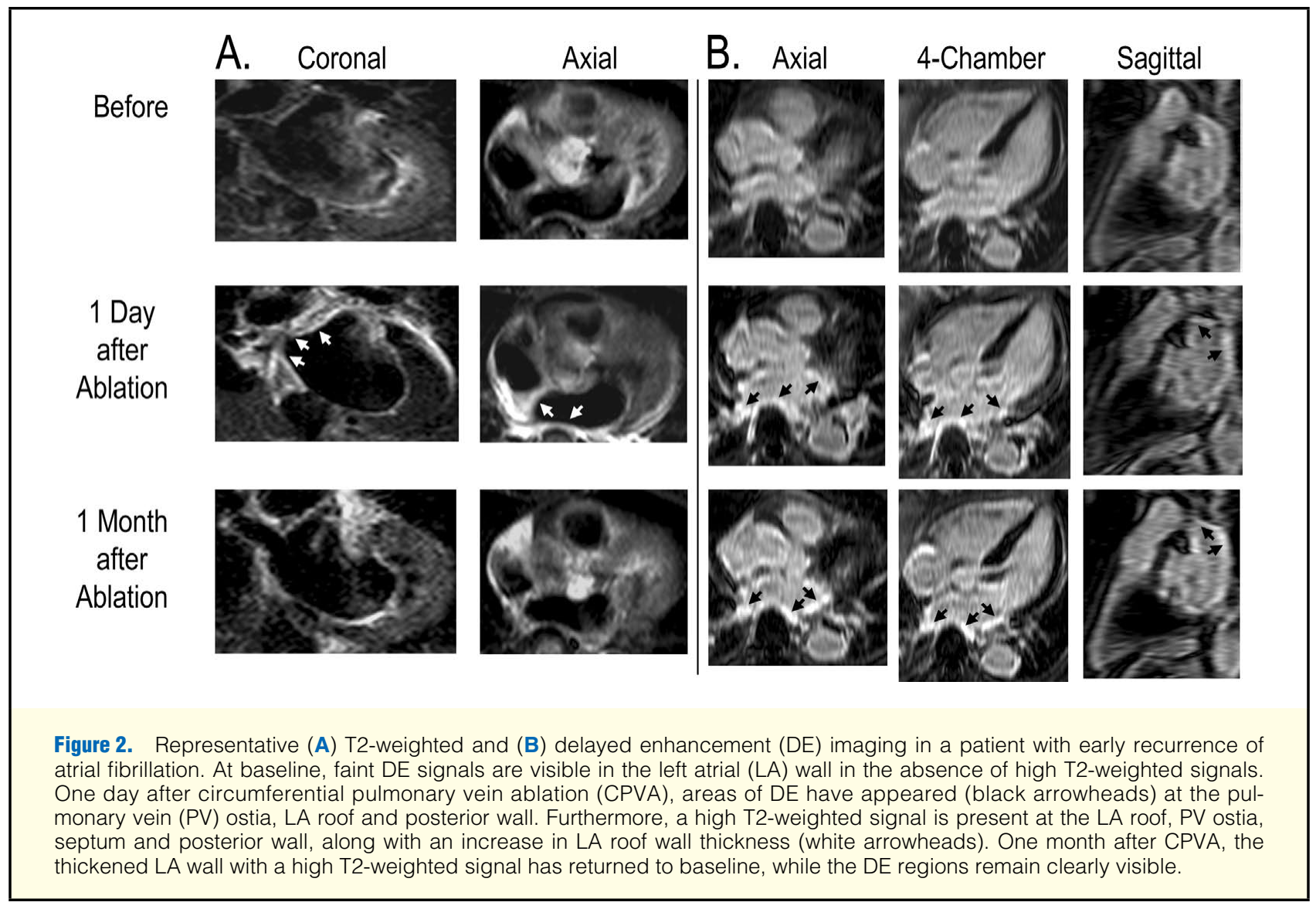

24-h ambulatory ECGs, or both, were recorded. The patients were investigated in detail, with the purpose of detecting the interim development of symptoms consistent with recurrence of arrhythmias. Recurrent AF was diagnosed when (1) patients reported symptoms suggestive of a tachycardia; and (2) a $>30$-s episode of AF was recorded on the 12-lead or 24-h ambulatory ECG. All episodes of AF that occurred within 1 month after the CPVA were classified as early recurrence of AF (ERAF).

\section{Statistical Analysis}

Continuous variables are expressed as mean \pm SD and were compared using Student's t-test. Analysis of variance was used when comparisons involved $>2$ groups. When significant between-group differences were detected, 1-way analysis of variance was followed by Scheffe's method to examine the significance of the differences among the means in all groups. Categorical variables were compared on chi-square test and Yate's correction, if necessary. Receiver operating characteristic (ROC) curves were constructed to examine the predictive value of the CMRI measurements with respect to the ERAFs. The optimal cut-off was identified as augmentation in the wall thickness associated with the greatest sum of the sensitivity and specificity. $\mathrm{P}<0.05$ was considered significant.

\section{Results}

\section{ERAF After Ablation}

All patients underwent uncomplicated catheter ablation of AF and successful electrical isolation of all identified PVs. At 


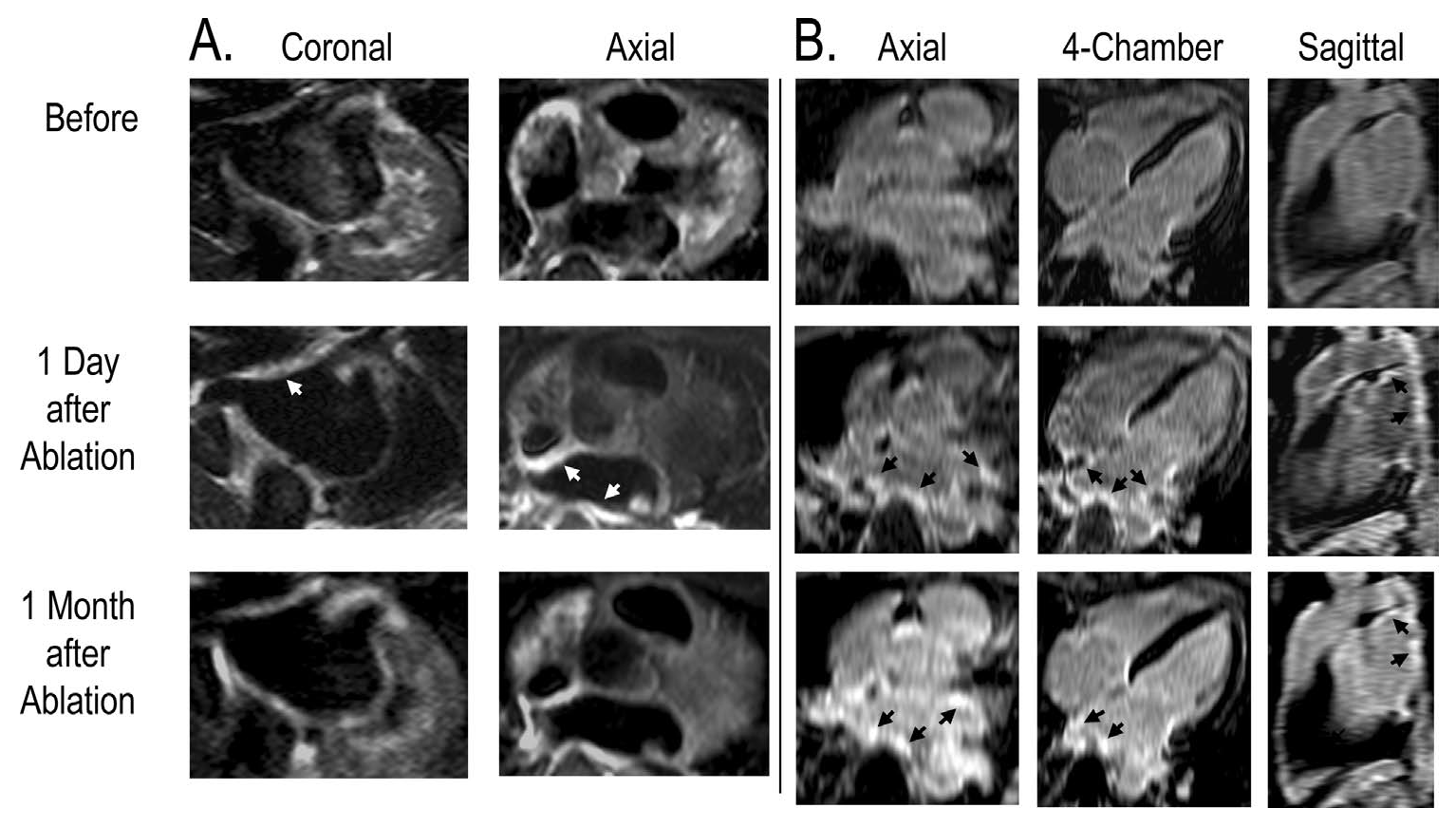

Figure 3. Representative (A) T2-weighted and (B) delayed enhancement (DE) imaging in a patient without an early recurrence of atrial fibrillation. One day after circumferential pulmonary vein ablation (CPVA), areas of DE are visible at the pulmonary vein ostia, left atrial (LA) roof, and posterior wall (black arrowheads). Although a high T2-weighted signal is present at the LA roof, septum and posterior wall (white arrowheads), the roof thickness is not increased. One month after CPVA, the thickened LA wall with a high T2-weighted signal has returned to baseline, and the DE regions remain visible.

a mean of $7 \pm 10$ days (range, 1-28 days) after the CPVA, 23 patients $(41 \%)$ developed 1 or more episodes of AF (ERAF group), while 33 patients (59\%) remained free from any $\mathrm{AF}$ during the first month of follow up (no-ERAF group). In the ERAF group, the first recurrence of AF occurred within $24 \mathrm{~h}$ after the CPVA in $33 \%$ of the patients, and within 1 week in $75 \%$. Except for a significantly higher proportion of men in the no-ERAF group, the baseline characteristics of the 2 study groups were similar (Table 1). Furthermore, no significant difference was observed between the 2 groups in terms of the total duration or total amount of RF energy delivered during the CPVA (Table 1).

\section{Thickness of LA Wall and T2-Weighted Imaging}

The measurement variables of the LA wall thickness and number of high-signal T2-weighted regions before the CPVA in all patients are given in Table 2. Before the CPVA, no region with a high T2-weighted signal was found in any patient from either group (Figures 2A,3A). There was no significant difference in the wall thickness among the 5 LA regions in either group or in each LA region between the 2 groups (Figure 4). One day after the CPVA, however, high T2-weighted signals were detected around the LA wall, most commonly in the LA roof $(100 \%)$, septum $(86 \%)$, posterior region $(71 \%)$, and PV ostia (Figures $\mathbf{2 A}, \mathbf{3 A}, \mathbf{5 A}$; Tables 1,2). In both groups, the mean wall thickness was significantly greater in all 5 LA regions 1 day after the ablation than at baseline (Figure 4; Table 2). Furthermore, the LA roof wall thickness $(\mathrm{P}<0.05)$ and its increase from baseline $(\mathrm{P}<0.0001)$ were greater in the ERAF group than in the no-ERAF group (Figure 4; Table 1).

The prevalence of high-signal T2-weighted regions significantly decreased 1 month after the CPVA in the LA roof (from $100 \%$ to $36 \%$; $\mathrm{P}<0.0001$ ), septum (from $86 \%$ to $54 \%$; $\mathrm{P}<0.05$ ) and posterior wall (from $71 \%$ to $7 \%$; $\mathrm{P}<0.0001$ ). After 1 month, the overall number of high-signal T2-weighted regions was significantly lower when compared to 1 day after the CPVA in both study groups (both $\mathrm{P}<0.05$; Figures $2 \mathrm{~A}, 3 \mathrm{~A}$, 5A). The LA wall thickness, at 1 month, returned to baseline and was similar in both study groups (Figures $2 \mathrm{~A}, 3 \mathbf{A}, \mathbf{4}$ ).

\section{DE of LA Wall Imaging}

Data on DE regions before the CPVA in all patients are given in Table 2. Although an LA wall signal was present before the CPVA, its intensity was distinctly lower than after the ablation procedure (Figures 2B,3B) and no difference was observed in the overall number of $\mathrm{DE}$ regions between the 2 groups at baseline $(\mathrm{P}=0.34$; Figure $5 \mathrm{~B})$. One day after the CPVA, the prevalence of DE regions significantly increased in the LA roof (from $50 \%$ to $100 \% ; \mathrm{P}<0.0001$ ), anterior wall (from $47 \%$ to $81 \% ; \mathrm{P}<0.01$ ), posterior wall (from $60 \%$ to $97 \%$; $\mathrm{P}<0.001$ ), septum (from $67 \%$ to $92 \% ; \mathrm{P}<0.05$ ) and lateral wall (from $67 \%$ to $94 \% ; \mathrm{P}<0.01$ ). The overall number of DE regions increased similarly and significantly in both groups (both $\mathrm{P}<0.05$; Figure 5B; Table 1 ). One month after the CPVA, clear contrast enhancement was present in the LA roof $(98 \%)$, anterior wall $(76 \%)$, posterior wall $(98 \%)$, septum (96\%), and lateral wall (94\%) on all scans (Figures 2B,3B). The overall number of DE regions was similar in both groups, as well as similar to the overall number of DE regions present 1 day after the CPVA (Figure 5B).

\section{Sensitivity and Specificity of Predictors of ERAF After Ablation}

On ROC analysis, a 1.15-mm increase in the LA roof thick- 


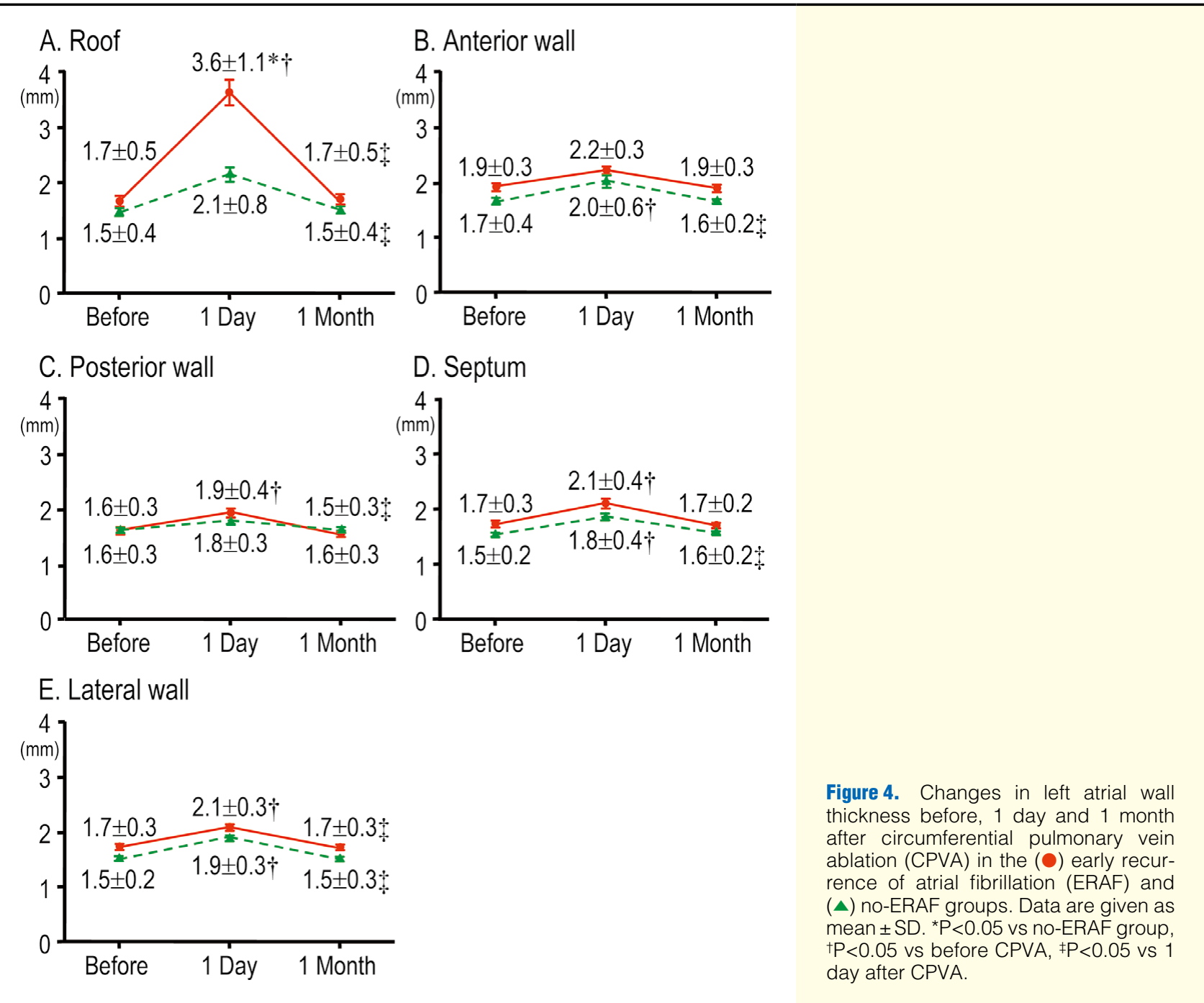

ness 1 day after the CPVA predicted an ERAF with an $85 \%$ sensitivity and $78 \%$ specificity.

\section{Recurrent AF 6 Months After Ablation and CMRI Findings}

At 6-month follow up, AF had recurred in 11 of the 56 patients $(20 \%)$. Anti-arrhythmic drugs were administered to 9 of 11 patients (82\%) with late recurrence of AF vs 26 of 45 patients $(58 \%)$ without $(\mathrm{P}=0.26)$. Furthermore, 6 of 23 patients $(26 \%)$ in the ERAF group developed late recurrence of AF vs 5 of 33 patients $(15 \%)$ in the no-ERAF group $(\mathrm{P}=0.50)$. In both the patients with and without late recurrence of $\mathrm{AF}$, the mean LA roof wall thickness was significantly greater 1 day after the ablation than at baseline (late-recurrence AF: from $1.6 \pm 0.4 \mathrm{~mm}$ to $2.9 \pm 1.6 \mathrm{~mm}$, no late-recurrence $\mathrm{AF}$ : from $1.4 \pm 0.3 \mathrm{~mm}$ to $2.9 \pm 1.2 \mathrm{~mm}$, both $\mathrm{P}<0.05)$. No significant difference was observed, however, in the mean LA roof wall thickness between the patients with vs those without late recurrence of $\mathrm{AF} 1$ day after the ablation $(\mathrm{P}=0.96)$.

\section{Discussion}

\section{Major Findings}

The main results of the present study are (1) CMRI detected the thickening and high T2-weighted signals that occurred in the LA wall shortly after the CPVA; (2) an increase in the LA roof thickness shortly after ablation was greater in the ERAF than in the no-ERAF group; (3) 1 month after the CPVA, the thickening and high T2-weighted signal had resolved, although the prevalence of DE regions remained unchanged in both groups; and (4) no significant difference was observed between the 2 groups in any of the CMRI characteristics before and 1 month after the CPVA. These observations indicate that the severity of the thickening associated with a high T2-weighted signal may predict an ERAF after CPVA in patients with paroxysmal AF, and that CMRI is useful for a detailed and periodic evaluation of LA tissue characteristics after CPVA.

\section{Thickening of LA Wall Shortly After CPVA}

Previous histopathology studies demonstrated that interstitial edema was found in the affected area of the myocardium $2 \mathrm{~h}$ after RF ablation, ${ }^{13}$ and that the endocardium and subendocardium displayed edematic loosening and microfragmentation of the connective tissue fibers shortly after ablation. ${ }^{14}$ Coagulation necrosis and interstitial hemorrhages also were found in the affected myocardium. ${ }^{13,15}$ All of those responses to the acute inflammation, especially the edema and hemorrhages, are responsible for the thickening of the myocardial 


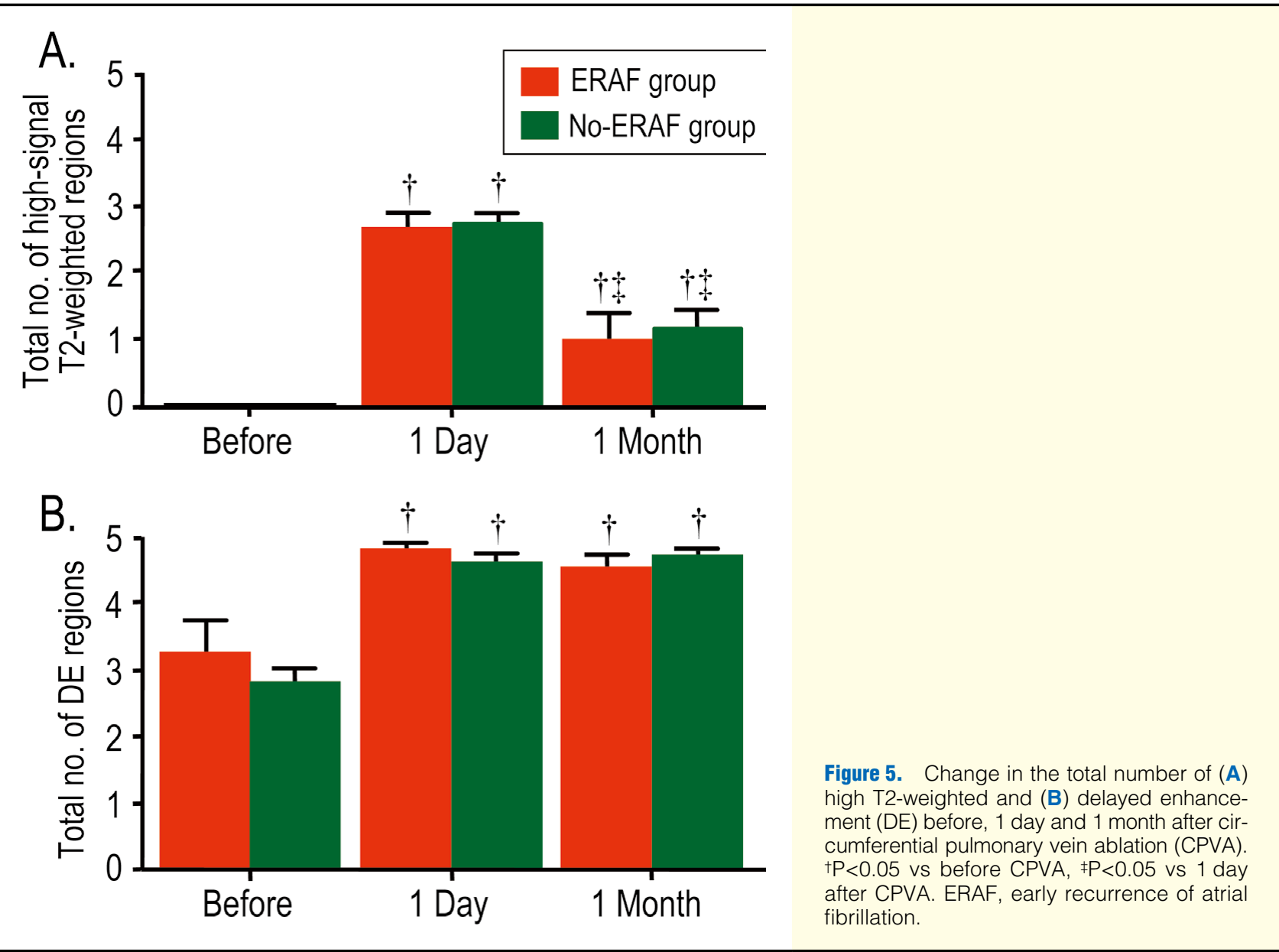

wall shortly after ablation. Recent studies have demonstrated that atrial wall thickening after ablation could occur within $15 \mathrm{~s}$ after RF energy delivery, ${ }^{8}$ and that it could resolve nearly completely within 1 month, ${ }^{4}$ which corresponds to the timing of the resolution of an acute inflammation response, especially with the edema, after the ablation. ${ }^{16,17}$

Wall thickening, which plays a part in the acute myocardial inflammatory response after ablation, was the basis for utilizing T2-weighted imaging. ${ }^{11,12}$ The present study has demonstrated that the acute inflammation responses to RF energy delivery were detected 1 day after the ablation as an increase in LA wall thickness, as shown by high T2-weighted signal, and their resolution 1 month after the ablation was confirmed by a thinning of the wall and decrease in the T2-weighted signal. The thickening has also been demonstrated to extend beyond the actual lesion within the atrium. These results obtained from the serial CMRI assessment were compatible with the previous studies using different imaging modalities, 4,8 and the changes in the atrial wall thickness were comparable to the previous histopathology. ${ }^{16,17}$ Therefore, we think that CMRI is useful for assessing and determining acute inflammation response after ablation.

\section{DE-CMRI of LA Edema and Scar After CPVA}

DE-CMRI has been used to image chronically scarred ventricular tissue in a variety of conditions, including old myocardial infarctions, ${ }^{18}$ myofibrillar disarray of hypertrophic cardiomyopathy, ${ }^{19,20}$ idiopathic scar from dilated cardiomyopathy, ${ }^{21,22}$ or scars of catheter ablation lesions. ${ }^{6,7}$ Recent studies have observed that despite considerable structural differences between acute and chronic myocardial infarctions, both tissues exhibited DE.11,12 DE is apparently due to the increased volume of the distribution of the gadolinium chelates secondary to the expansion of the extracellular space in acute myocardial infarctions and in old myocardial scars. ${ }^{12}$ In an acute myocardial infarction, the loss of the membrane integrity of the edematous cardiomyocytes allows communication between the extra- and intracellular spaces. ${ }^{12}$ In contrast, in a chronic myocardial infarction, enlargement of the extracellular space is mostly the product of a relatively large collagen matrix in the absence of myocardial edema. ${ }^{12}$ The mechanism underlying the enlargement of the extracellular space after the CPVA in the present study may explain the high prevalence of DE regions in the LA wall 1 day and 1 month after the CPVA. Even if the underlying mechanism of this extracellular space enlargement is different, the distinction between edematous and scar tissue, using DE-CMRI only, is challenging. We believe that for a detailed CMRI assessment of the wall thickness, DE and T2-weighted imaging are key for periodic examinations and the precise characterization of the LA myocardium after ablation.

\section{Predictors of ERAF and Thickening of LA Roof}

Several predictors of an ERAF have been reported, including age, LA diameter, number of AF foci, and successful or unsuccessful anatomical ablation. ${ }^{8}$ While acute inflammatory changes after ablation may be responsible for an immediate recurrence of $\mathrm{AF},{ }^{23,24}$ no procedural complications occurred 
in the present study that might have caused a diffuse inflammatory response. A recent study found that acute inflammatory changes due to RF energy is a cause of ERAF, ${ }^{5}$ and the changes associated with RF ablation and the slow development of scar tissue are thought to be physiologic correlates of ERAFs. ${ }^{15-17}$ In the present study, an increase in the wall thickness, as shown by high T2-weighted signal, was observed shortly after ablation in nearly all patients. Increase in LA roof thickness, however, was significantly greater among the patients with than without ERAFs, and it predicted an ERAF. A previous study using human hearts showed that the LA roof was the thinnest and the posterior wall the second thinnest region, ${ }^{25}$ and that lesions created in the thin myocardial layer regularly exhibited complete transmural coagulation necrosis after RF ablation. ${ }^{17}$ Furthermore, the favorable anatomic relationship between the site of the trans-septal puncture and the LA roof facilitated the placement of the ablation catheter in close contact and with optimal pressure exerted against the myocardium. This may have caused a greater degree of inflammation and edematous changes in the LA roof after the CPVA and, subsequently, a higher incidence of ERAFs.

\section{Study Limitations}

First, we did not examine the factors associated with inflammation, such as C-reactive protein, body temperature and others in the acute phase following RF ablation. Therefore, the relationship between the changes in these variables and an ERAF, or CMRI findings, is unknown. Second, DE-CMRI for detecting LA wall scar is challenging because compared with the left ventricle, the wall is thin. Furthermore, the measurement might not be the actual thickness of the LA wall. Because of the limited spatial resolution of MRI, the LA myocardium could not be distinguished from the interstitial space and epicardium, before as well as after the CPVA. In a pig model, however, Schwartzman et al measured a 0-mm thick interstitial space before ablation, vs an interstitial edema thickness equal to approximately $50-60 \%$ of the right atrial wall thickness after linear ablation of the right atrium. ${ }^{16}$ Therefore, we believe that the change in the LA wall thickness, although not entirely, was mainly due to the edema of the LA myocardium. Third, the previous studies used 3-dimensional (D) DE-CMRI to identify and quantify the LA wall scar 13 months after RF ablation. ${ }^{6,7}$ DE-CMRI also facilitated the correction with 3-D CARTO images and demonstrated a tissue injury pattern reflecting the location of the RF energy delivery sites. ${ }^{7}$ But, we used conventional 2-D CMRI, and the present methodology for the assessment of the DE and highsignal T2-weighted regions was semi-quantitative. The ablation was performed under fluoroscopic guidance. Therefore, the precise relationship between the ablation sites and regions of thickening is unclear. Fourth, we did not systematically monitor the patients' rhythm after their discharge from hospital, unless they developed symptoms suggestive of recurrent tachyarrhythmias. Therefore, asymptomatic recurrences may have remained undetected. Fifth, women were a majority in the ERAF group. In a previous study of 221 patients, however, the early procedural success rate was similar in men and women. ${ }^{26}$ In addition, we observed no significant genderrelated differences in the CMRI changes after CPVA (data not shown). Therefore, this gender-related difference might be attributable to the small size of the patient sample. Finally, we did not meticulously compare, between the 2 groups, the differences in the RF energy delivered to the various LA regions.

\section{Acknowledgments}

We are indebted to the radiological and medical technologists at Gunma Prefectural Cardiovascular Center for their important contribution to the present study.

\section{Disclosure}

The authors have no conflict of interest to disclose.

\section{References}

1. Oral H, Scharf C, Chugh A, Hall B, Cheung P, Good E, et al. Catheter ablation for paroxysmal atrial fibrillation: Segmental pulmonary vein ostial ablation versus left atrial ablation. Circulation 2003; 108: $2355-2360$.

2. Verma A, Kilicaslan F, Pisano E, Marrouche NF, Fanelli R, Brachmann J, et al. Response of atrial fibrillation to pulmonary vein antrum isolation is directly related to resumption and delay of pulmonary vein conduction. Circulation 2005; 112: 627-635.

3. Li XP, Dong JZ, Liu XP, Long de Y, Yu RH, Tian Y, et al. Predictive value of early recurrence and delayed cure after catheter ablation for patients with chronic atrial fibrillation. Circ J 2008; 72: $1125-1129$.

4. Okada T, Yamada T, Murakami Y, Yoshida N, Ninomiya Y, Shimizu $\mathrm{T}$, et al. Prevalence and severity of left atrial edema detected by electron beam tomography early after pulmonary vein ablation. $J$ Am Coll Cardiol 2007; 49: 1436-1442.

5. Oral H, Knight BP, Ozaydin M, Tada H, Chugh A, Hassan S, et al. Clinical significance of early recurrences of atrial fibrillation after pulmonary vein isolation. J Am Coll Cardiol 2002; 40: 100-104.

6. Peters DC, Wylie JV, Hauser TH, Kissinger KV, Botnar RM, Essebag V, et al. Detection of pulmonary vein and left atrial scar after catheter ablation with three-dimensional navigator-gated delayed enhancement MR imaging: Initial experience. Radiology 2007; 243: 690-695.

7. McGann CJ, Kholmovski EG, Oakes RS, Blauer JJ, Daccarett M, Segerson N, et al. New magnetic resonance imaging-based method for defining the extent of left atrial wall injury after the ablation of atrial fibrillation. J Am Coll Cardiol 2008; 52: 1263-1271.

8. Weerasooriya R, Jaïs P, Sanders P, Scavée C, Hsu LF, Hocini M, et al. Images in cardiovascular medicine. Early appearance of an edematous tissue reaction during left atrial linear ablation using intracardiac echo imaging. Circulation 2003; 108: e80.

9. Simonetti OP, Finn JP, White RD, Laub G, Henry DA. 'Black blood' T2-weighted inversion-recovery MR imaging of the heart. Radiology 1996; 199: 49-57.

10. Essebag V, Baldessin F, Reynolds MR, McClennen S, Shah J, Kwaku KF, et al. Non-inducibility post-pulmonary vein isolation achieving exit block predicts freedom from atrial fibrillation. Eur Heart J 2005; 26: 2550-2555.

11. Saeed M, Weber O, Lee R, Do L, Martin A, Saloner D, et al. Discrimination of myocardial acute and chronic (scar) infarctions on delayed contrast enhanced magnetic resonance imaging with intravascular magnetic resonance contrast media. J Am Coll Cardiol 2006; 48: $1961-1968$.

12. Abdel-Aty H, Zagrosek A, Schulz-Menger J, Taylor AJ, Messroghli $\mathrm{D}$, Kumar A, et al. Delayed enhancement and T2-weighted cardiovascular magnetic resonance imaging differentiate acute from chronic myocardial infarction. Circulation 2004; 109: 2411-2416.

13. Schwartzman D, Parizhskaya M, Devine WA. Linear ablation using an irrigated electrode electrophysiologic and histologic lesion evolution comparison with ablation utilizing a non-irrigated electrode. J Interv Card Electrophysiol 2001; 5: 17-26.

14. Deneke T, Khargi K, Müller KM, Lemke B, Mügge A, Laczkovics A, et al. Histopathology of intraoperatively induced linear radiofrequency ablation lesions in patients with chronic atrial fibrillation. Eur Heart J 2005; 26: 1797-1803.

15. Tanno K, Kobayashi Y, Kurano K, Kikushima S, Yazawa T, Baba $\mathrm{T}$, et al. Histopathology of canine hearts subjected to catheter ablation using radiofrequency energy. Jpn Circ J 1994; 58: 123-135.

16. Schwartzman D, Ren JF, Devine WA, Callans DJ. Cardiac swelling associated with linear radiofrequency ablation in the atrium. J Interv Card Electrophysiol 2001; 5: 159-166.

17. Taylor GW, Kay GN, Zheng X, Bishop S, Ideker RE. Pathological effects of extensive radiofrequency energy applications in the pulmonary veins in dogs. Circulation 2000; 101: 1736-1742.

18. Wu E, Judd RM, Vargas JD, Klocke FJ, Bonow RO, Kim RJ. Visualization of presence, location, and transmural extent of healed Q-wave and non-Q-wave myocardial infarction. Lancet 2001; 357: 
$21-28$.

19. Choudhury L, Mahrholdt H, Wagner A, Choi KM, Elliott MD, Klocke FJ, et al. Myocardial scarring in asymptomatic or mildly symptomatic patients with hypertrophic cardiomyopathy. J Am Coll Cardiol 2002; 40: 2156-2164.

20. Motoyasu M, Kurita T, Onishi K, Uemura S, Tanigawa T, Okinaka $\mathrm{T}$, et al. Correlation between late gadolinium enhancement and diastolic function in hypertrophic cardiomyopathy assessed by magnetic resonance imaging. Circ $J$ 2008; 72: 378-383.

21. Mahrholdt H, Wagner A, Judd RM, Sechtem U, Kim RJ. Delayed enhancement cardiovascular magnetic resonance assessment of nonischaemic cardiomyopathies. Eur Heart J 2005; 26: 1461 - 1474.

22. Cho JR, Park S, Choi BW, Kang SM, Ha JW, Chung N, et al. Delayed enhancement magnetic resonance imaging is a significant prognostic factor in patients with non-ischemic cardiomyopathy. Circ J 2010; 3: 476-483.
23. Lee SH, Tai CT, Hsieh MH, Tsai CF, Lin YK, Tsao HM, et al. Predictors of early and late recurrence of atrial fibrillation after catheter ablation of paroxysmal atrial fibrillation. J Interv Card Electrophysiol 2004; 10: 221-226.

24. Koyama T, Sekiguchi Y, Tada H, Arimoto T, Yamasaki H, Kuroki $\mathrm{K}$, et al. Comparison of characteristics and significance of immediate versus early versus no recurrence of atrial fibrillation after catheter ablation. Am J Cardiol 2009; 103: 1249-1254.

25. Hall B, Jeevanantham V, Simon R, Filippone J, Vorobiof G, Daubert J. Variation in left atrial transmural wall thickness at sites commonly targeted for ablation of atrial fibrillation. J Interv Card Electrophysiol 2006; 17: $127-132$.

26. Forleo GB, Tondo C, De Luca L, Dello Russo A, Casella M, De Sanctis V, et al. Gender-related differences in catheter ablation of atrial fibrillation. Europace 2007; 9: 613-620. 